\title{
A novel GATA6 mutation associated with congenital ventricular septal defect
}

\author{
GUI-FEN ZHENG $^{1 *}$, DONG WEI ${ }^{*}$, HONG ZHAO ${ }^{1}$, NING ZHOU $^{1}$, YI-QING YANG ${ }^{2}$ and XING-YUAN LIU ${ }^{1}$ \\ ${ }^{1}$ Department of Pediatrics, Tongji Hospital, Tongji University School of Medicine, Shanghai 200065; \\ ${ }^{2}$ Department of Cardiovascular Research, Shanghai Chest Hospital, Medical College of \\ Shanghai Jiaotong University, Shanghai 200030, P.R. China
}

Received December 29, 2011; Accepted February 6, 2012

DOI: $10.3892 / \mathrm{ijmm} .2012 .930$

\begin{abstract}
Ventricular septal defect (VSD) is the most common form of congenital cardiovascular malformation and an important contributor to the substantially increased morbidity and mortality in infants. Emerging evidence indicates the genetic basis for the pathogenesis of congenital VSD in a significant proportion of patients. However, congenital VSD is a genetically heterogeneous disease and the genetic defects responsible for VSD in the overwhelming majority of cases remain unclear. In this study, the entire coding region of the GATA6 gene, which encodes a zinc-finger transcription factor crucial to normal cardiogenesis, was sequenced in 130 unrelated patients with congenital VSD. The available relatives of the index patient carrying the identified mutation and 200 unrelated ethnically matched healthy individuals used as controls were subsequently genotyped. The functional characteristics of the mutant GATA6 were assessed in contrast to its wild-type counterpart using a luciferase reporter assay system. As a result, a novel heterozygous missense GATA6 mutation, p.G220S, was identified in a proband with VSD. The variation was absent in 400 control chromosomes and the altered amino acid was highly conserved evolutionarily across species. Genetic analysis of the family members of the mutation carrier showed that the substitution co-segregated with VSD was inherited as an autosomal dominant trait. Functional analysis demonstrated that the p.G220S mutation of GATA6 was associated with significantly decreased transcriptional activity. The findings provide novel insight into the molecular mechanism involved in VSD, implying the potential clinical implications in the gene-specific prophylaxis and therapy of this common developmental abnormality in neonates.
\end{abstract}

Correspondence to: Dr Xing-Yuan Liu, Department of Pediatrics, Tongji Hospital, Tongji University School of Medicine, 389 Xin Cun Road, Shanghai 200065, P.R. China

E-mail: liuxingyuan789@gmail.com

*Contributed equally

Key words: ventricular septal defect, transcription factor, genetics

\section{Introduction}

Congenital heart disease is the most common type of developmental deformity, affecting approximately 1 in 20 live births, 1 in 100 newborn infants that require medical interventions during the first year of life, and is the major noninfectious cause of child mortality, accounting for one third of deaths due to birth defects (1-3). Congenital cardiovascular abnormalities are clinically classified into at least 18 distinct categories with many additional anatomic variations, of which ventricular septal defect (VSD) is the most prevalent form, occurring in nearly $50 \%$ of all children with congenital cardiovascular anomalies, and in $14-16 \%$ of cardiac malformations that require an invasive treatment within one year of age $(3,4)$. Congenital VSD may occur alone or together with other cardiovascular deformations, such as atrial septal defect, pulmonary artery stenosis, or tetralogy of Fallot. Irrespective of other defects that may accompany VSD, isolated VSD may result in cardiac enlargement, congestive heart failure, pulmonary hypertension, Eisenmenger's syndrome, delayed fetal brain development, arrhythmias, and even sudden cardiac death in the absence of surgical or catheterbased repair (5-9). Despite the important clinical significance, the molecular etiology giving rise to VSD in the vast majority of the patients remains largely unknown $(10,11)$.

Development of the heart is a complex biological process involving multiple molecular pathways that modulate cardiac morphogenesis. Disruption of cardiac development at any point during primary embryogenesis may lead to structural cardiac defects, including VSD (4). Recent studies in developmental biology have demonstrated that cardiac transcription factors, including GATA family members, play a pivotal role in primary heart development (12-14). GATA transcription factors are characterized by their ability to bind to the consensus DNA sequence 'GATA'. In vertebrates, six members of the GATA family have been identified, of which GATA4, GATA5 and GATA6 are expressed broadly in the heart and endodermallyderived tissues $(15,16)$. Of these three GATA transcription factors, GATA4 has been most extensively explored and is believed to be essential for cardiogenesis (17). Presently, a long list of GATA4 mutations have been identified in patients with a wide variety of diseases, including VSD, atrial septal defects, atrioventricular septal defects, tetralogy of Fallot, pulmonary stenosis, patent ductus arteriosus, and atrial fibrillation (18-32). 
GATA6 is another member of the GATA family with expression and functions that partially overlap with GATA4 during cardiac embryogenesis (15). Emerging evidence underlines the key role of GATA6 in cardiovascular morphogenesis (33-36), hence suggesting the potential involvement of GATA6 mutations in the pathogenesis of structural congenital cardiovascular aberrations. Although mutations of GATA6 have recently been implicated with persistent truncus arteriosus, tetralogy of Fallot, atrial septal defects, and atrioventricular septal defects, the association of GATA6 mutations with VSD is still not well established (37-39).

To assess the prevalence and spectrum of GATA6 mutations responsible for VSD, the coding exons and exon/intron boundaries of GATA6 were sequenced in a cohort of 130 unrelated cases with congenital VSD, and a novel heterozygous GATA6 mutation, p.G220S, was identified in a VSD patient with positive family history. Subsequently, genetic analysis of the mutation carrier's family members available showed that the mutation co-segregated with VSD and was inherited in an autosomal dominant pattern. Functional analysis demonstrated that the p.G220S mutation of GATA6 was associated with significantly decreased transcriptional activity. These findings provide novel insight into the genetic mechanisms involved in human heart development.

\section{Materials and methods}

Study participants. A cohort of 130 unrelated patients with VSD was identified among the Chinese Han population. Subjects were evaluated by individual and familial history, review of the medical records, complete physical examination, 12-lead electrocardiogram (ECG) and two-dimensional transthoracic echocardiography with color flow Doppler. All patients had a classic form of VSD, with a defect diameter of $>3 \mathrm{~mm}$ and nearly all patients underwent cardiac catheterization and, if required, cardiac surgery. The types of VSD were defined using two-dimensional continuous wave Doppler and color Doppler techniques on transthoracic echocardiography. Where necessary, transesophageal echocardiography and angiography were used for further clarification of the anatomy. A subarterial VSD was distinguished from a perimembranous VSD by color Doppler flow mapping and confirmed by direct view during the surgical procedure. A total of 200 ethnically matched unrelated healthy individuals were recruited as controls to screen for the identified mutation in GATA6. Peripheral venous blood samples from VSD patients and control individuals were prepared. The study protocol was reviewed and approved by the local institutional ethics committee and written informed consent was obtained from all participants or their guardians prior to the study.

Genetic studies. Genomic DNA from all participants was extracted from blood lymphocytes with the Wizard Genomic DNA Purification kit (Promega, Madison, WI, USA). The candidate gene GATA6 was screened initially in 130 unrelated patients with VSD and genotyping GATA6 in the available relatives of the index patient harboring an identified mutation and the 200 control individuals was subsequently performed. The referential genomic DNA sequence of GATA6 was derived from GenBank (accession no. NT_010966). The primer pairs used to amplify the whole coding region of GATA6 by polymerase chain reaction (PCR) were designed as shown in Table I. PCR was conducted using HotStar TaqDNA Polymerase (Qiagen, Hilden, Germany) on a PE 9700 Thermal Cycler (Applied Biosystems, Foster City, CA, USA), with standard conditions and concentrations of reagents. Amplified products were analyzed on $1 \%$ agarose gels stained with ethidium bromide and purified with the QIAquick Gel Extraction kit (Qiagen). Both strands of each PCR product were sequenced with a BigDye $^{\circledR}$ Terminator v3.1 Cycle Sequencing kit (Applied Biosystems) under an ABI PRISM 3130XL DNA Analyzer (Applied Biosystems). The sequencing primers were the same as previously designed for specific region amplification. The DNA sequences were viewed and analyzed with the DNA Sequencing Analysis Software v5.1 (Applied Biosystems). The variant was validated by resequencing an independent PCR-generated amplicon from the subject and met our quality control thresholds with a call rate of $>99 \%$.

Multiple sequence alignments. The multiple GATA6 protein sequences across various species were aligned using the online software of ClustalW2 (an interactive program at http://www. ebi.ac.uk/Tools/msa/clustalw2/).

Prediction of the disease-causing potential of a GATA6 sequence variation. The disease-causing potential of a GATA6 sequence variation was predicted automatically by Mutation Taster (an online program at http://www.mutationtaster.org), giving a probability for the alteration to be either a causative mutation or a harmless polymorphism. Notably, the P-value used here is the probability of the prediction rather than the probability of error as used in t-test statistics, i.e. a value close to 1 indicates a high 'security' of the prediction.

Plasmids and site-directed mutagenesis. The recombinant expression plasmid pcDNA3-hGATA6 was kindly provided by Dr Angela Edwards-Ghatnekar, from the Division of Rheumatology and Immunology, Medical University of South Carolina, Charleston, SC, USA. The atrial natriuretic peptide (ANP)-luciferase reporter gene, which contains the 2600-bp 5 -flanking region of the ANP gene, namely ANP(-2600)-Luc, was kindly provided by Dr Ichiro Shiojima, from the Department of Cardiovascular Science and Medicine, Chiba University Graduate School of Medicine, Chuo-ku, Chiba, Japan. The identified mutation was introduced into the wild-type GATA6 using a QuikChange II XL Site-Directed Mutagenesis kit (Stratagene, La Jolla, CA, USA) with a complementary pair of primers. The mutant was sequenced to confirm the desired mutation and to exclude any other sequence variations.

Reporter gene assays. HEK-293 cells were cultured in Dulbecco's modified Eagle's medium supplemented with $10 \%$ fetal calf serum. The ANP(-2600)-Luc reporter construct and an internal control reporter plasmid pGL4.75 (hRluc/CMV, Promega) were used in transient transfection assays to examine the transcriptional activation function of the GATA6 mutant. HEK-293 cells were transfected with $0.4 \mu \mathrm{g}$ of wild-type or mutant pcDNA3hGATA6 expression vector, $0.4 \mu \mathrm{g}$ of ANP(-2600)-Luc reporter construct, and $0.04 \mu \mathrm{g}$ of pGL4.75 control reporter vector using PolyFect Transfection Reagent (Qiagen). For co-transfection experiments, $0.2 \mu \mathrm{g}$ of wild-type pcDNA3-hGATA6, $0.2 \mu \mathrm{g}$ 
Table I. Intronic primers used to amplify the coding exons and exon-intron boundaries of GATA6.

\begin{tabular}{|c|c|c|c|}
\hline Exon & Forward primer & Reverse primer & Amplicon (bp) \\
\hline $2-\mathrm{a}$ & $5^{\prime}$-ttgttaacccgtcgatctcc-3' & $5^{\prime}$-gcgagggtctggtacatctc-3' & 543 \\
\hline $2-b$ & $5^{\prime}$-tgctgttcactgacctcgac-3' & 5'-ctgggagagtaggggaagc-3' & 466 \\
\hline $2-c$ & 5'-ccgacagccetccatacg-3' & 5'-gaaaacagggcccgagtg-3' & 539 \\
\hline 3 & 5'-ggccaaggagaaaagctcag-3' & 5'-gttggaacagccgggacag-3' & 485 \\
\hline 4 & 5'-tcttggcccagaaaagtcag-3' & 5'-tcatttgctgattctttgtaactg-3' & 387 \\
\hline 5,6 & 5'-ctgggattagaggcgtgagc-3' & 5'-tttactagagagcagcccagt-3' & 473 \\
\hline 7 & $5^{\prime}$-atttctcctgccetgggtct-3' & 5'-ctgcacaaaagcagacacga-3' & 382 \\
\hline
\end{tabular}

Table II. Clinical characteristics of the 130 unrelated patients with congenital ventricular septal defects.

\begin{tabular}{|c|c|c|}
\hline & $\mathrm{N}$ & Percentage or range \\
\hline Male & 58 & 45 \\
\hline Age at the diagnosis of VSD (years) & $3.66^{\mathrm{a}}$ & $0.25-15$ \\
\hline Age at the present study (years) & $5.87^{\mathrm{a}}$ & $0.50-18$ \\
\hline Positive family history & 14 & 11 \\
\hline \multicolumn{3}{|l|}{ Distribution of different types of VSDs } \\
\hline Subpulmonary & 10 & 8 \\
\hline Perimembranous & 95 & 73 \\
\hline Atrioventricular canal & 9 & 7 \\
\hline Muscular & 16 & 12 \\
\hline \multicolumn{3}{|l|}{ Prevalence of VSDs with other defects } \\
\hline Isolated VSD & 98 & 75 \\
\hline VSD and ASD & 14 & 11 \\
\hline VSD and ASD and PDA & 8 & 6 \\
\hline VSD and ASD and DORV & 5 & 4 \\
\hline VSD and PDA & 3 & 2 \\
\hline VSD and PS & 2 & 2 \\
\hline \multicolumn{3}{|l|}{ Incidence of arrhythmias } \\
\hline Atrioventricular block & 5 & 4 \\
\hline Atrial fibrillation & 2 & 2 \\
\hline \multicolumn{3}{|l|}{ Treatment } \\
\hline Surgical repair & 103 & 79 \\
\hline Percutaneous closure & 12 & 9 \\
\hline Follow-up & 15 & 12 \\
\hline
\end{tabular}

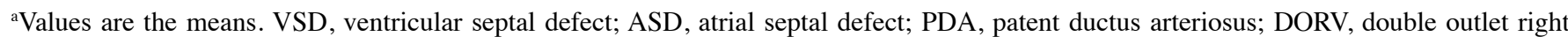
ventricle; PS, pulmonary stenosis.

of mutant pcDNA3-hGATA6, $0.4 \mu \mathrm{g}$ of ANP(-2600)-Luc, and $0.04 \mu \mathrm{g}$ of pGL4.75 were used. Firefly luciferase and Renilla luciferase activities were measured with the Dual-Glo luciferase assay system (Promega) $48 \mathrm{~h}$ after transfection. Three independent experiments were performed at a minimum for wild-type and mutant GATA6.

Statistics. Data are given as mean \pm SD. Differences between the two groups were compared with Student's t-test for continuous variables and a 2 -tailed $\mathrm{P}$-value $<0.05$ was considered to be statistically significant.

\section{Results}

Characteristics of the study subjects. A cohort of 130 unrelated patients with VSD was registered and clinically evaluated and contrasted to a total of 200 ethnically matched unrelated healthy individuals used as controls. None of them had overt traditional risk factors for VSD. The baseline clinical characteristics of the 130 unrelated patients with VSD are summarized in Table II.

GATA6 mutations. Direct sequencing of the coding exons and flanking introns of the GATA6 gene was performed after 


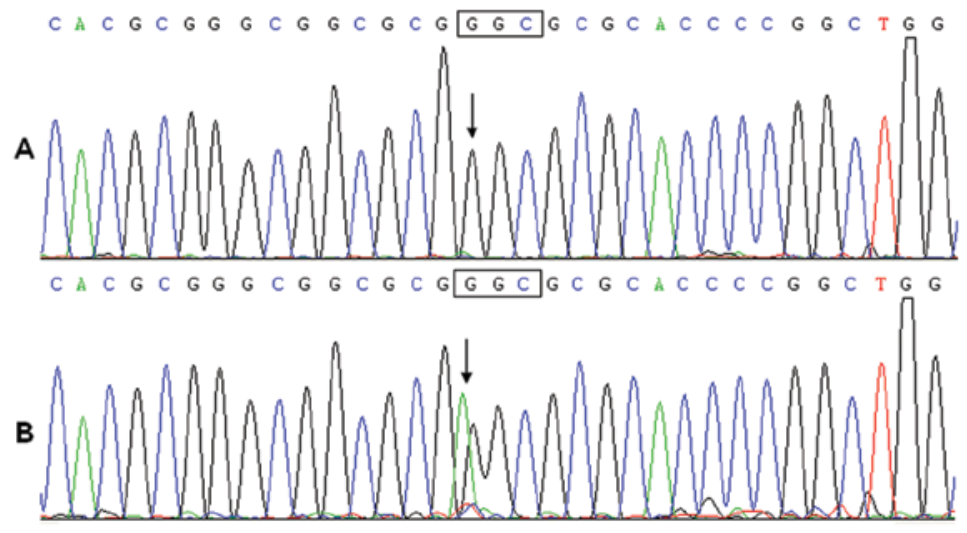

Figure 1. Representative sequence chromatograms showing the GATA6 mutation compared with the control sequence. The arrow indicates (B) the heterozygous nucleotides of A/G in the proband from family 1 or (A) the homozygous nucleotides of $\mathrm{G} / \mathrm{G}$ in a control individual. The rectangle denotes the nucleotides comprising a codon of GATA6.

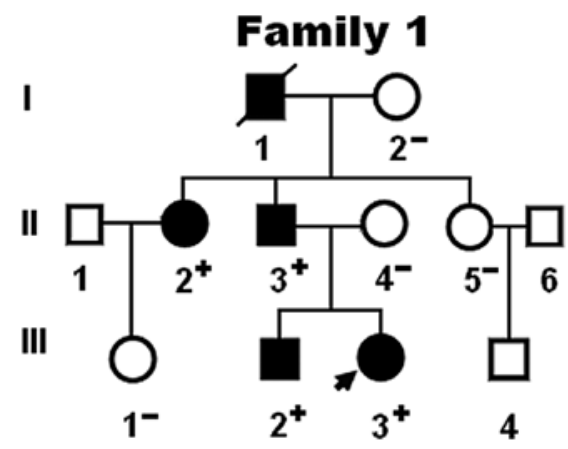

Figure 2. Pedigree structure of the family with congenital ventricular septal defect. Family members are identified by generations and numbers. Squares indicate male family members; circles, female members; symbols with a slash, the deceased members; closed symbols, affected members; open symbols, unaffected members; arrow, proband; + , carriers of the heterozygous mutation; and -, non-carriers.

PCR amplification of genomic DNA from the 130 unrelated VSD patients. A heterozygous substitution of adenine (A) for guanine $(\mathrm{G})$ in the first nucleotide of codon 220 of the GATA6 gene (c.658G $>A$ ), predicting the transition of glycine to serine at amino acid position 220 (p.G220S), was identified in 1 out of 130 patients. The total population prevalence of GATA6 mutation based on the cohort patients was approximately $0.77 \%$. The representative sequence chromatograms showing the detected heterozygous GATA6 variation of c. $658 \mathrm{G}>\mathrm{A}$ compared with the control sequence are shown in Fig. 1. The variant was neither detected in the control population nor reported in the SNP database at the website http://www.ncbi. nlm.nih.gov/SNP. Genetic scan of the relatives of the mutation carrier displayed that the gene variant was present in all affected family members alive, but absent in unaffected family members examined. Analysis of the pedigree demonstrated that the mutation co-segregated with VSD transmitted in an autosomal dominant fashion in the family with complete penetrance. The pedigree structure of the family is illustrated in Fig. 2. The phenotypic characteristics and results of genetic screening of the affected pedigree members are listed in Table III. All the 4 living patients underwent surgical repair of the VSD.
Multiple alignments of the GATA6 protein sequences across species. A cross-species alignment of GATA6 protein sequences displayed that the altered amino acid G220 was highly conserved evolutionarily as shown in Fig. 3, suggesting that the amino acid is functionally important.

Disease-causing potential of a GATA6 sequence variation. The sequence variation of c.658G $>\mathrm{A}$ detected in GATA6 was automatically predicted to be disease-causing with a P-value of 0.530867 , providing evidence for the alteration to be a malicious disease mutation rather than a benign polymorphism. No SNPs in the altered region were found in the MutationTaster database.

Transcriptional activity of the GATA6 mutant. The transcriptional activation function of GATA6 in HEK-293 cells was investigated using one of its direct cardiac downstream target genes, $A N P$, as a luciferase reporter, and the activity of the $A N P$ promoter was presented as fold activation of firefly luciferase relative to Renilla luciferase. The same amounts of wildtype $(0.4 \mu \mathrm{g})$ and mutant GATA6 $(0.4 \mu \mathrm{g})$ activated the ANP promoter by $\sim 11.9$-fold and $\sim 5.7$-fold, respectively. When the same amount of wild-type GATA6 $(0.2 \mu \mathrm{g})$ was cotransfected with mutant GATA6 $(0.2 \mu \mathrm{g})$, the induced activation of the ANP promoter was $\sim 7.4$-fold. These results suggest that mutant GATA6 has significantly reduced activation activity compared with wild-type GATA6 (Fig. 4).

\section{Discussion}

In the present study, we report a previously unrecognized missense mutation of p.G220S in GATA6 identified in a family with congenital VSD. The novel heterozygous mutation was present in all the affected family members alive but absent in unaffected relatives examined and in the 400 normal chromosomes from a matched control population. A cross-species alignment of GATA6 protein sequences showed that the altered amino acid was evolutionarily highly conserved. The p.G220S variant was predicted to be pathogenic by MutationTaster, and the functional analysis substantiated that the p.G220S-mutant GATA6 was associated with significantly decreased transcriptional activity. Therefore, it is very likely that dysfunctional GATA6 is responsible for VSD in this family. 
Table III. Phenotypic characteristics and status of the GATA6 mutation in the affected pedigree members.

\begin{tabular}{|c|c|c|c|c|c|c|c|}
\hline \multicolumn{4}{|c|}{ Subject information } & \multicolumn{3}{|c|}{ Phenotype } & \multirow{2}{*}{$\frac{\text { Genotype }}{\text { G220S }}$} \\
\hline Identity & Gender & $\begin{array}{l}\text { Age at time of study } \\
\text { (years) }\end{array}$ & $\begin{array}{c}\text { Age at diagnosis of VSD } \\
\text { (years) }\end{array}$ & $\begin{array}{l}\text { VSD } \\
(\mathrm{mm})\end{array}$ & Other cardiac defects & AVB & \\
\hline $\mathrm{I}-1$ & M & $58^{\mathrm{a}}$ & 43 & 22 & PS & + & N/A \\
\hline II-2 & $\mathrm{F}$ & 36 & 9 & 9 & - & - & $+/-$ \\
\hline II-3 & M & 31 & 7 & 7 & ASD & - & $+/-$ \\
\hline III-2 & M & 6 & 4 & 11 & - & - & $+/-$ \\
\hline III-3 & $\mathrm{F}$ & 2 & 2 & 5 & - & - & $+/-$ \\
\hline
\end{tabular}

${ }^{\mathrm{a}}$ Age of death. F, female; M, male; PS, pulmonary stenosis; VSD, ventricular septal defect; AVB, atrioventricular block; ASD, atrial septal defect; N/A, not available or not applicable; +, indicates present; and -, denotes absent.

\begin{tabular}{|c|c|c|}
\hline & & \\
\hline Human (NP_005248.2) & 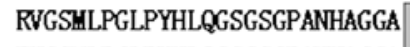 & \\
\hline Monkey (XP_002800933.1) & RVGSWLPGLPYHLQGSGSGPANHAGGA & G AHPGPP-----QASADSPPYGGGG \\
\hline Dog (XP_547642.3) & RVGSWLPGLPY-LQGAGGGPANHAGGA & G KHPGVPFHEGDFVRVDVSVPPRSS \\
\hline $\mathrm{Ca}$ & RANKMLF & 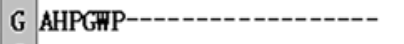 \\
\hline 99493.2) & RVGSWLPGLPY-LC & G SHPGHP-----QA \\
\hline Mouse (NP_C34388.2) & RVGSWLSGLPY-LQGAGSGPSNHAGGA & G AHPGWS-----QASADSPPYG-GG \\
\hline -062058.1) & RVGSMLPGLPY-LQGAGSGPSNHAGGA & G AHPGWP-----QASADSPPYG-GG \\
\hline Fowl(NP_990751.1) & RVGSVLPTLPY-LQGGGAAQPGHAP-A & G HVWSQP-------AAESPSYGAAG \\
\hline Frog (NAAP82292.1) & RVGSHLTSISYLQG-TGASQGAHSVNN & H HSQATS----------EGSSFSSSS \\
\hline Zebrafish(NP_571632.1) & RVGTMIPNLSYLHRRYQHSRARVSSHS & SQPAP---------ESPSYSTGS \\
\hline
\end{tabular}

Figure 3. Alignment of multiple GATA6 protein sequences across species. The altered amino acid of p.G220 is highly evolutionarily conserved among species.

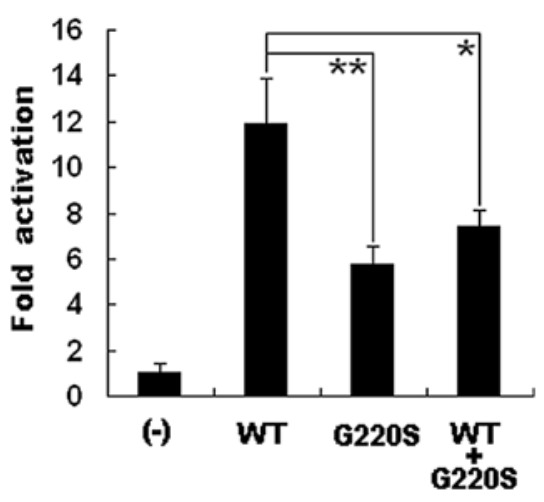

Figure 4. Decreased transcriptional activity of the mutant GATA6 on ANP promoter. Activation of ANP-luciferase reporter in HEK-293 cells by GATA6 wild type (WT), mutant (G220S), alone or in combination, demonstrated significantly decreased transactivation by mutant protein. Experiments were performed in triplicate and mean and standard deviations are shown. ${ }^{*} \mathrm{P}<0.05$ and ${ }^{* *} \mathrm{P}<0.01$, respectively, when compared with the wild type GATA6.

In humans the GATA6 gene maps to chromosome 18q11.1q11.2, encoding a protein composed of 449 amino acids (17). Topologically GATA6 contains a transcriptional activation domain essential for its transcriptional activity, a zinc finger domain required for DNA sequence recognition and binding to the consensus motif, and a nuclear localization signal associated with the subcellular trafficking and distribution of GATA6 (17). The GATA6 mutation of p.G220S identified in this study is located in the transcriptional activation domain, thus may be expected to exert direct influence on the transcriptional activity of GATA6.

Previous studies have revealed that GATA6 is an upstream transcriptional regulator of several genes expressed during cardiac development, including genes that encode atrial natriuretic peptide, brain natriuretic factor, gap junction protein, and $\beta$-myosin heavy chain (40). GATA6 may bind to downstream target DNA alone or together with other partners including NKX2-5, GATA4, GATA5 and TBX5, and the synergistic transcriptional activations mediated by these transcription factors have been substantiated $(36,41,42)$. Therefore, the functional effect of the GATA6 mutation may be evaluated by biochemical analysis of the transcriptional activity of the ANP promoter in cells transfected with mutant GATA6 in contrast to the wild-type counterpart. In this study, the functional properties of the novel p.G220S mutation of GATA6 identified in our VSD patients was explored by transcriptional activity assays and the results showed significantly decreased transcriptional activity on a downstream gene, consistent with the loss-of function effect of another GATA6 mutation underlying congenital atrial septal defect and tetralogy of Fallot on the transcriptional activity of the ANP promoter (38). These findings imply that haploinsufficiency or loss-of-function effect arising from GATA6 mutation is potentially an alternative pathophysiological mechanism involved in congenital VSD.

To date, 5 distinct GATA6 mutations identified in 7 of 601 patients with a spectrum of cardiovascular defects have 
been reported, with a mutation prevalence of $1.16 \%$ (37-39). Kodo et al (37) sequenced the GATA6 gene in 21 unrelated patients with persistent truncus arteriosus and 2 different heterozygous mutations of p.E486GfsX10 and p.N466H were identified in 2 patients, respectively, with a prevalence of $9.52 \%$. It is of note that the proband harboring the frameshift mutation also had atrial septal defect, his father and sister carried the identical mutation, but his father only had pulmonary stenosis and his sister suffered from pulmonary stenosis, atrial septal defect, and patent ductus arteriosus. Functional analysis showed that both GATA6 mutations caused defects in nuclear localization and transactivation activity. Lin et al (38) carried out systematic genetic analysis of GATA6 in 270 unrelated patients with congenital heart diseases and found a heterozygous mutation of p.S184N in 3 patients, including 1 with tetralogy of Fallot and 2 with atrial septal defect, with a prevalence of $1.11 \%$. Biological analysis revealed that the mutation had clearly reduced transcriptional activity. Maitra et al (39) genotyped GATA6 in 310 unrelated patients with congenital cardiovascular diseases and 2 heterozygous mutations of p.A178V and p.L198V were identified in 2 patients, respectively, with a prevalence of $0.65 \%$. The L198V variant carrier was affected with tetralogy of Fallot whereas the A178V variant carrier was affected with atrioventricular septal defect, hypoplastic left ventricle, and VSD. Functional assays showed that the A178V mutation had increased transcriptional activity while the L198V mutation had no impact on the transcriptional activity (39). Similarly, a GATA6 mutation prevalence of $0.77 \%$ $(1 / 130)$ was observed in our VSD population, implying that the GATA6 mutations could be a minor cause of congenital VSD. Additionally, the proband's father carrying the p.G220S mutation had also atrial septal defect. Genetic background, unknown second modifiers, epigenetic factors, and environmental factors might account for the phenotypic variability within the families mentioned above.

It is noteworthy that striking genetic heterogeneity of VSD was highlighted by an inability to find mutations in about $99 \%$ of our cohort patients, despite somatic GATA6 mutations as a potential etiology of VSD in some patients. Therefore, the contribution of genes other than GATA6 to VSD pathogenesis seems certain. Considering that GATA and NK transcription factors cooperatively and synergistically regulate downstream cardiac genes and there is a threshold for these factors to function properly in heart development $(36,41,42)$, we also screened the GATA4 and NKX2-5 genes in the 130 unrelated patients with VSD as previously described (29-32,43-45). However no non-synonymous mutations were detected, excluding the possibility of these 2 transcription factor deficits involved in the pathogenesis of VSD in this study.

Association of functionally deficient GATA6 with increased susceptibility to cardiac abnormalities has been substantiated in animals. Homozygous GATA6 null mice die after implantation because of defects in visceral endoderm function and extraembryonic development $(35,46)$. Although mice that are heterozygous for GATA4 or GATA6 null mutations are viable and without obvious cardiovascular phenotypes, mice that are compound heterozygous for both GATA4 and GATA6 die by E13.5 with $100 \%$ penetrance, displaying a spectrum of cardiovascular defects, including VSD, persistent truncus arteriosis, myocardial hypoplasia, reduced myocardial proliferation, and impaired differentiation of vascular smooth muscle cells (33). Similarly, compound loss of a GATA5 and a GATA6 allele also leads to double outlet right ventricle and VSD in mice (42). These findings demonstrate an exquisite sensitivity of the developing cardiovascular system to the levels of GATA4, GATA5 and GATA6 and suggest that these GATA factors act cooperatively to regulate downstream target genes.

The discovery of a novel GATA6 mutation in patients with congenital VSD aids in an early and precise diagnosis, which is clinically important, because leaving it uncorrected until adulthood may lead to congestive heart failure and pulmonary hypertension, although some patients with minor VSD can often live an otherwise healthy life. As the mortality rate of VSD operations is very low in patients without cardiac failure at an early age, timely surgical repair or catheter-based closure of VSD is feasible. This information will be very useful for genetic counseling for this family, because genetic testing for p.G220S in new family members can be followed by careful medical examination and prophylactic intervention if VSD is observed.

In conclusion, this study links a novel loss-of-function mutation in the cardiac transcription factor GATA6 to VSD, which provides new insight into the molecular mechanism involved in the pathogenesis of VSD, and suggests the potential clinical implications for the gene-specific prophylaxis and therapy of this common disease in children.

\section{Acknowledgements}

We are greatly indebted to participants for their dedication to the study. This study was supported in part by grants from the Natural Science Fund of Shanghai, China (10ZR1433100 and 10ZR1428000) and the National Natural Science Fund of China (81070153 and 30570768).

\section{References}

1. Hoffman JI and Kaplan S: The incidence of congenital heart disease. J Am Coll Cardiol 39: 1890-1900, 2002.

2. Hoffman JI, Kaplan S and Liberthson RR: Prevalence of congenital heart disease. Am Heart J 147: 425-439, 2004.

3. Roger VL, Go AS, Lloyd-Jones DM, Adams RJ, Berry JD, Brown TM, Carnethon MR, Dai S, de Simone G, Ford ES, et al; American Heart Association Statistics Committee and Stroke Statistics Subcommittee: Heart disease and stroke statistics - 2011 update: a report from the American Heart Association. Circulation 123: e18-e209, 2011.

4. Minette MS and Sahn DJ: Ventricular septal defects. Circulation 114: 2190-2197, 2006.

5. Sommer RJ, Hijazi ZM and Rhodes JF Jr: Pathophysiology of congenital heart disease in the adult: part I: shunt lesions. Circulation 117: 1090-1099, 2008.

6. McQuillen PS and Miller SP: Congenital heart disease and brain development. Ann NY Acad Sci 1184: 68-86, 2010.

7. Walsh EP: Interventional electrophysiology in patients with congenital heart disease. Circulation 115: 3224-3234, 2007.

8. Walsh EP and Cecchin F: Arrhythmias in adult patients with congenital heart disease. Circulation 115: 534-545, 2007.

9. Yap SC and Harris L: Sudden cardiac death in adults with congenital heart disease. Expert Rev Cardiovasc Ther 7: 1605-1620, 2009.

10. Jenkins KJ, Correa A, Feinstein JA, Botto L, Britt AE, Daniels SR, Elixson M, Warnes CA and Webb CL; American Heart Association Council on Cardiovascular Disease in the Young: Noninherited risk factors and congenital cardiovascular defects: current knowledge: a scientific statement from the American Heart Association Council on Cardiovascular Disease in the Young: endorsed by the American Academy of Pediatrics. Circulation 115: 2995-3014, 2007. 
11. Pierpont ME, Basson CT, Benson DW Jr, Gelb BD, Giglia TM, Goldmuntz E, McGee G, Sable CA, Srivastava D and Webb CL; American Heart Association Congenital Cardiac Defects Committee, Council on Cardiovascular Disease in the Young: Genetic basis for congenital heart defects: current knowledge: a scientific statement from the American Heart Association Congenital Cardiac Defects Committee, Council on Cardiovascular Disease in the Young: endorsed by the American Academy of Pediatrics. Circulation 115: 3015-3038, 2007.

12. Pikkarainen S, Tokola H, Kerkelä R and Ruskoaho H: GATA transcription factors in the developing and adult heart. Cardiovasc Res 63: 196-207, 2004.

13. Nemer M: Genetic insights into normal and abnormal heart development. Cardiovasc Pathol 17: 48-54, 2008

14. Benson DW: Genetic origins of pediatric heart disease. Pediatr Cardiol 31: 422-429, 2010.

15. Molkentin JD: The zinc finger-containing transcription factors GATA-4, -5 , and -6 . Ubiquitously expressed regulators of tissuespecific gene expression. J Biol Chem 275: 38949-38952, 2000

16. Patient RK and McGhee JD: The GATA family (vertebrates and invertebrates). Curr Opin Genet Dev 12: 416-422, 2002.

17. Brewer A and Pizzey J: GATA factors in vertebrate heart development and disease. Expert Rev Mol Med 8: 1-20, 2006.

18. Garg V, Kathiriya IS, Barnes R, Schluterman MK, King IN, Butler CA, Rothrock CR, Eapen RS, Hirayama-Yamada K, Joo K, et al: GATA4 mutations cause human congenital heart defects and reveal an interaction with TBX5. Nature 424: 443-447, 2003

19. Okubo A, Miyoshi O, BabaK, Takagi M,Tsukamoto K, Kinoshita A, Yoshiura K, Kishino T, Ohta T, Niikawa N and Matsumoto N: A novel GATA4 mutation completely segregated with atrial septal defect in a large Japanese family. J Med Genet 41: e97, 2004.

20. Reamon-Buettner SM and Borlak J: GATA4 zinc finger mutations as a molecular rationale for septation defects of the human heart. J Med Genet 42: e32, 2005.

21. Hirayama-Yamada K, Kamisago M, Akimoto K, Aotsuka H, Nakamura Y, Tomita H, Furutani M, Imamura S, Takao A, Nakazawa M and Matsuoka R: Phenotypes with GATA4 or NKX2.5 mutations in familial atrial septal defect. Am J Med Genet A 135: 47-52, 2005

22. Nemer G, Fadlalah F, Usta J, Nemer M, Dbaibo G, Obeid M and Bitar F: A novel mutation in the GATA4 gene in patients with Tetralogy of Fallot. Hum Mutat 27: 293-294, 2006.

23. Tomita-Mitchell A, Maslen CL, Morris CD, Garg V and Goldmuntz E: GATA4 sequence variants in patients with congenital heart disease. J Med Genet 44: 779-783, 2007.

24. Rajagopal SK, Ma Q, Obler D, Shen J, Manichaikul A, TomitaMitchell A, Boardman K, Briggs C, Garg V, Srivastava D, et al Spectrum of heart disease associated with murine and human GATA4 mutation. J Mol Cell Cardiol 43: 677-685, 2007.

25. Zhang W, Li X, Shen A, Jiao W, Guan X and Li Z: GATA4 mutations in 486 Chinese patients with congenital heart disease. Eur J Med Genet 51: 527-535, 2008.

26. Hamanoue H, Rahayuningsih SE, Hirahara Y, Itoh J, Yokoyama U, Mizuguchi T, Saitsu H, Miyake N, Hirahara F and Matsumoto N Genetic screening of 104 patients with congenitally malformed hearts revealed a fresh mutation of GATA4 in those with atrial septal defects. Cardiol Young 19: 482-485, 2009.

27. Chen MW, Pang YS, Guo Y, Pan JH, Liu BL, Shen J and Liu TW: GATA4 mutations in Chinese patients with congenital cardiac septal defects. Pediatr Cardiol 31: 85-89, 2010.

28. Chen Y, Mao J, Sun Y, Zhang Q, Cheng HB, Yan WH, Choy KW and Li H: A novel mutation of GATA4 in a familial atrial septal defect. Clin Chim Acta 411: 1741-1745, 2010.

29. Liu XY, Wang J, Zheng JH, Bai K, Liu ZM, Wang XZ, Liu X, Fang WY and Yang YQ: Involvement of a novel GATA4 mutation in atrial septal defects. Int J Mol Med 28: 17-23, 2011.
30. Wang J, Fang M, Liu XY, Xin YF, Liu ZM, Chen XZ, Wang XZ, Fang WY, Liu X and Yang YQ: A novel GATA4 mutation responsible for congenital ventricular septal defects. Int J Mol Med 28: 557-564, 2011.

31. Jiang JQ, Shen FF, Fang WY, Liu X and Yang YQ: Novel GATA4 mutations in lone atrial fibrillation. Int J Mol Med 28: 1025-1032, 2011.

32. Yang YQ, Wang MY, Zhang XL, Tan HW, Shi HF, Jiang WF, Wang XH, Fang WY and Liu X: GATA4 loss-of-function mutations in familial atrial fibrillation. Clin Chim Acta 412: 1825-1830, 2011.

33. Xin M, Davis CA, Molkentin JD, Lien CL, Duncan SA, Richardson JA and Olson EN: A threshold of GATA4 and GATA6 expression is required for cardiovascular development. Proc Natl Acad Sci USA 103: 11189-11194, 2006.

34. Holtzinger A and Evans T: Gata5 and Gata6 are functionally redundant in zebrafish for specification of cardiomyocytes. Dev Biol 312: 613-622, 2007.

35. Zhao R, Watt AJ, Battle MA, Li J, Bondow BJ and Duncan SA: Loss of both GATA4 and GATA6 blocks cardiac myocyte differentiation and results in acardia in mice. Dev Biol 317: 614-619, 2008.

36. Maitra M, Schluterman MK, Nichols HA, Richardson JA, Lo CW, Srivastava D and Garg V: Interaction of Gata4 and Gata6 with Tbx 5 is critical for normal cardiac development. Dev Biol 326: 368-377, 2009.

37. Kodo K, Nishizawa T, Furutani M, Arai S, Yamamura E, Joo K, Takahashi T, Matsuoka R and Yamagishi H: GATA6 mutations cause human cardiac outflow tract defects by disrupting semaphorinplexin signaling. Proc Natl Acad Sci USA 106: 13933-13938, 2009

38. Lin X, Huo Z, Liu X, Zhang Y, Li L, Zhao H, Yan B, Liu Y, Yang Y and Chen YH: A novel GATA6 mutation in patients with tetralogy of Fallot or atrial septal defect. J Hum Genet 55: 662-667, 2010.

39. Maitra M, Koenig SN, Srivastava D and Garg V: Identification of GATA6 sequence variants in patients with congenital heart defects. Pediatr Res 68: 281-285, 2010.

40. Rémond MC, Iaffaldano G, O'Quinn MP, Mezentseva NV, Garcia V, Harris BS, Gourdie RG, Eisenberg CA and Eisenberg LM: GATA6 reporter gene reveals myocardial phenotypic heterogeneity that is related to variations in gap junction coupling. Am J Physiol Heart Circ Physiol 301: H1952-H1964, 2011.

41. Zhang Y, Rath N, Hannenhalli S, Wang Z, Cappola T, Kimura S, Atochina-Vasserman E, Lu MM, Beers MF and Morrisey EE: GATA and Nkx factors synergistically regulate tissue-specific gene expression and development in vivo. Development 134 : 189-198, 2007.

42. Laforest B and Nemer M: GATA5 interacts with GATA4 and GATA6 in outflow tract development. Dev Biol 358: 368-378, 2011.

43. Wang J, Xin YF, Liu XY, Liu ZM, Wang XZ and Yang YQ: A novel NKX2-5 mutation in familial ventricular septal defect. Int J Mol Med 27: 369-375, 2011.

44. Liu XY, Wang J, Yang YQ, Zhang YY, Chen XZ, Zhang W, Wang XZ, Zheng JH and Chen YH: Novel NKX2-5 mutations in patients with familial atrial septal defects. Pediatr Cardiol 32: 193-201, 2011.

45. Wang J, Liu XY and Yang YQ: Novel NKX2-5 mutations responsible for congenital heart disease. Genet Mol Res 10: 2905-2915, 2011.

46. Morrisey EE, Tang Z, Sigrist K, Lu MM, Jiang F, Ip HS and Parmacek MS: GATA6 regulates HNF4 and is required for differentiation of visceral endoderm in the mouse embryo. Genes Dev 12: 3579-3590, 1998. 